\title{
Impact of Neurotransmitters on Health through Emotion
}

\author{
Ms. G. Deepika ${ }^{1 *}$, Ms. Rajeswari. $\mathrm{H}^{2}$
}

Keywords: Neurotransmitters, Health, Emotion

Animal and Human behaviour suggests that it is ultimately an expression of events guided by the nervous system. As decision-making neurological processes became increasingly concentrated in the centralized brain over evolutionary time, behaviour became essentially an expression of brain's response to information coming to it from inside and outside the body.

Human emotions guide and direct behaviour. They dominate us in such a way that there is no solution. If a person has no emotions, he becomes crippled in terms of life. An emotion is a strong feeling associated with some instincts or biological drives. In addition to the above, emotions have some more specific characteristics-they bring psychological and physiological changes. Emotions are short-lived experiences that produce coordinated changes in people's thoughts, actions and physiological responses. During emotions, specific action tendencies infuse both mind and body, simultaneously narrowing individual action urges (flight in fear, attack in anger) by mobilizing appropriate bodily support for those specific actions.

Human emotions are classified into Negative (Fear, Anger, Depression, Anxiety, Envy, Shame etc.) and Positive (Love, Appreciation, Happiness, Hope, Confidence, Patience, Trust etc.) emotions. The primary emotions are anger, fear, pleasure, sadness, and disgust. Positive emotions are often characterized by a relative lack of autonomic reactivity. They broaden the scopes of attention, cognition, and action widening the array of precepts, thoughts and actions presently in mind. The positive emotion of pleasure may facilitate injective, exploratory, sexual or novel-seeking behaviour. Negative emotions such as anger and fear may promote avoidance or defensive behaviour. They may lead to Change in appetite, Headaches, High blood pressure, Insomnia, Sexual problems, Weight gain or loss, Chest pain. Studies have shown that negative emotions actually weaken your body, while positive emotions strengthen your body. Shame has the most devastating effect, followed by guilt, apathy, grief, fear, anxiety, craving, anger and

\footnotetext{
${ }^{1}$ Assistant Professor, Department Of Mental Health Nursing, Narayana College Of Nursing, Chinthareddypalem, Nellore

2 Professor \& HOD, Department Of Mental Health Nursing, Narayana College Of Nursing, Chinthareddypalem, Nellore

*Responding Author

(C) 2016 I G Deepika, H Rajeswari; licensee IJIP. This is an Open Access Research distributed under the terms of the Creative Commons Attribution License (http://creativecommons.org/licenses/by/2.0), which permits unrestricted use, distribution, and reproduction in any Medium, provided the original work is properly cited.
} 
hate. Negative emotions really do cause disease and illness and premature aging. Powerful emotions such as pain, fear, grief, disappointment, panic, anxiety, anger and longing shock your body like an electrical charge, leaving scars or lesions along your neural pathways.

\section{Neurotransmitters:}

Neurotransmitters are a chemical substance that acts as a mediator for the transmission of nerve impulse from one neuron to other neuron through a synapse. It is produced in the cell body of the neuron and is transported through axon. At the axon terminal, the neurotransmitter is stored in small packets called vesicles. Under the influence of stimulus, these vesicles open and release the neurotransmitter into synaptic cleft. It binds to the specific receptors on the surface of post synaptic cell and is responsible for the various actions produced. In short, neurotransmitters are the way nerve cells communicate with each other and with other cells in the body. Neurotransmitters are used to relay information about environment to the brain, to analyze the information and to set in motion appropriate bodily responses.

Most neurotransmitters can activate multiple receptor subtypes and receptor classes. If they were allowed to operate over a long period of time, the results would be disastrous for the organism since there would be a constant overload of messages being sent. Approximately neurotransmitters regulate human body functioning and contribute to normal functioning .They function by changing the permeability of the cell membrane to various ions such as sodium and potassium. If an excess of sodium ions flow into the nerve cell, an impulse is generated. If an excess of potassium ions flow out, the impulse is inhibited. Depending upon their function, neurotransmitters are classified into two types. Excitatory neurotransmitters and Inhibitory neurotransmitters. Excitatory neurotransmitters are responsible for the conduction of impulse from presynaptic neuron to postsynaptic neuron. Neurotransmitter released from pre synaptic axon terminal causes some change in resting membrane potential, i.e. slight depolarization by the opening of sodium channels in the postsynaptic membrane and influx of sodium ions from extra cellular fluid. This slight depolarization is called excitation. 


\section{Process of neurotransmission}

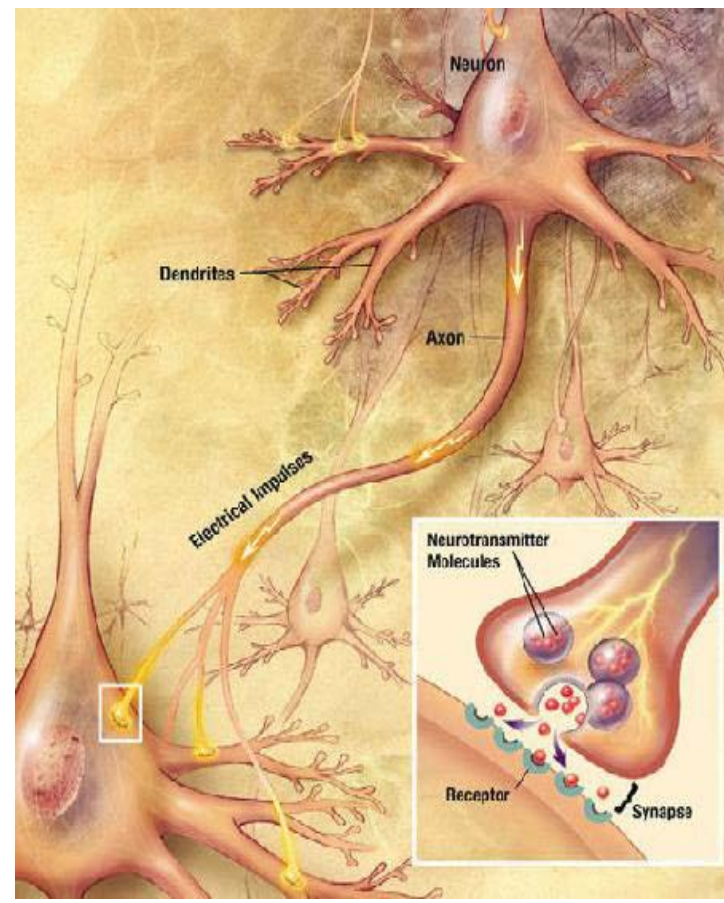

Common excitatory neurotransmitters are acetylcholine, nor adrenaline, glutamate, aspartate, histamine and nitric oxide. Inhibitory neurotransmitters inhibit the conduction of impulse from the pre synaptic neuron to postsynaptic neuron. When it is released from presynaptic axon terminal due to the arrival of action potential, it causes the release of potassium in the post synaptic membrane and efflux of potassium ions which leads to hyper polarization, also called as inhibition. Inhibitory neurotransmitters calm the brain and help create balance in mood. Common inhibitory neurotransmitters are dopamine, gamma amino butyric acid (GABA), glycine and serotonin. Different neurons, in different regions of the brain and carrying out different functions, may express different receptors for the same neurotransmitter. This allows the same neurotransmitter to affect neurons in different ways, depending on the type of receptor they display. Each receptor, when occupied, triggers a different kind of reaction within the receiving neuron. All neurotransmitters play some role in behaviour. The neurotransmitters most commonly implicated in behaviour modulation are the small molecular transmitters - acetyl choline, nor epinephrine, dopamine and serotonin. 


\section{Scheme of neurotransmission:}

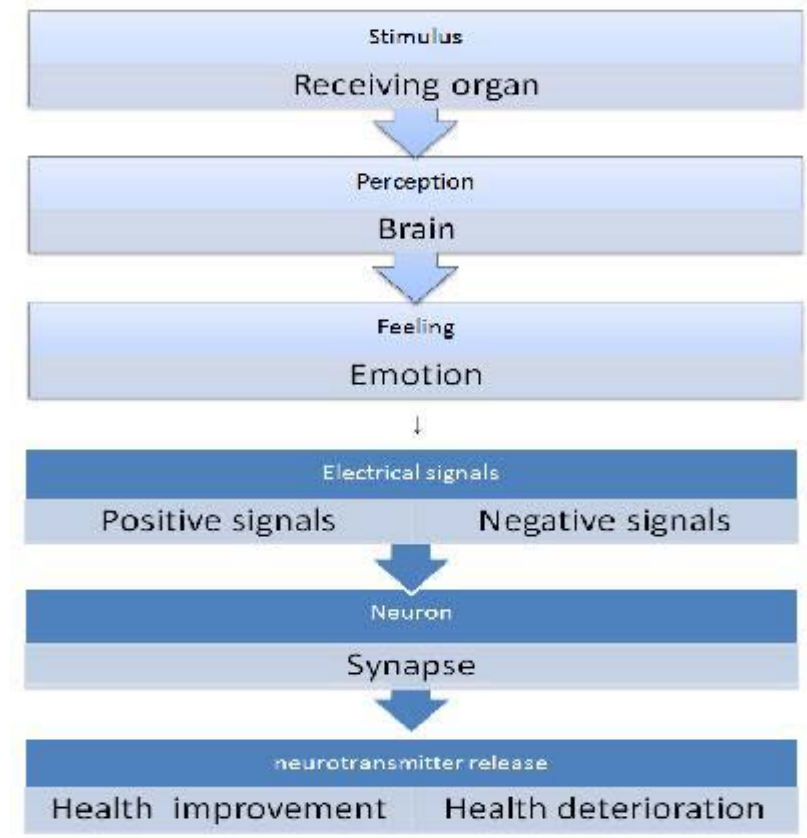

Acetylcholine (ACH): ACH is responsible for much of the stimulation of muscles, including the muscles of the gastro-intestinal system. It is transmitted within cholinergic pathways that are concentrated mainly in specific regions of the brainstem and are thought to be involved in cognitive functions, especially memory. Severe damage to these pathways is the probable cause of Alzheimer's disease. Outside the brain, acetylcholine is the main neurotransmitter in the parasympathetic nervous system - the system that controls functions such as heart rate, digestion, secretion of saliva and bladder function. It causes emotion, reward perception and long term depression. It plays an important role in memory formation.

\section{Serotonin :}

Serotonin has an unusually large number of different receptors present on various cells in the brain; a total of 15 receptors, spread across different structural classes. There are two presynaptic receptors that are important to behaviour - the serotonin transporter and a regulatory receptor referred to as the serotonin- $1 \beta$ receptor.

Adequate amounts of serotonin are necessary for a stable mood and to balance any excessive excitatory neurotransmitter firing in the brain. In addition to mood control, serotonin has been linked with a wide variety of functions, including the regulation of sleep, pain perception, body temperature, blood pressure and hormonal activity.

Activities such as eating, grooming, or simply resting and thinking are accompanied by high levels of brain serotonin. But it is also the main chemical messenger used to wake up the cortex and get it involved in decision making. In this role, serotonin is used largely by neurons found in the so-called "raphae nucleus" of the midbrain region, where possible behavioural responses to 


\section{Impact of Neurotransmitters on Health through Emotion}

various stimuli are first formulated. These neurons in turn become involved in the future processing and interpretation of the incoming messages, and in selecting an appropriate response.

Low levels of 5-HT and metabolites are associated with depression and especially a type of depression that is more likely to lead to suicide. Serotonin dysfunction has been associated with obsessive compulsive disorder, aggression, eating disorders, and schizophrenia and migraine headaches. Reduced serotonin may induce insomnia and decreased immune system functioning.

The decreased level of serotonin generated by the action of enzyme mono aminooxidase A is associated with violent behaviour and antisocial personality disorder. Increased level of serotonin during early life, caused by decreased activity of this enzyme, seems to be linked with the higher risk of violent behaviour and aggression in the adulthood.

\section{Histamine}

Histamine plays role in arousal, phobias, addictions and extreme depression, pain threshold, and regulation of blood pressure

\section{Noradrenaline (NA)}

NA is involved in a broad range of psychological functions and behaviours. One of the most important is its role in attention and arousal. It regulates anxiety and negative emotional memory reward perception. NA neurons appear to be involved in the regulation of an organism's vigilance. The broad projection of the locus coeruleus (LC) makes it especially well suited to act as a mechanism to alert cortical and thalamic areas to incoming sensory stimuli. The LC is electro-physiologically quiet during low vigilance states such as sleep or in the lack of sensory input. When exposed to a strong stimulus, the LC markedly increases its firing rate, however. The broad influence of the activated LC is to filter weak stimuli and enhance moderate stimuli. This filtering and enhancement by NA is believed to aid in CNS processing of sensory information.

Noradrenaline alerts the brain of the presence of novel and potentially threatening events in the external environment- brain arousal and body arousal. Under activity of noradrenaline-releasing neurons often accompanies depression. Over production of noradrenalin may generate feelings of anxiety and fearfulness, as if there was a constant threat present in the environment.

\section{Dopamine:}

The role of DA systems in motivated behaviour is of particular importance. It is proposed to mediate a performance activating effect of motivated behaviour, as well as conveying internal reward signals. DA is implicated in psychiatric illnesses (especially schizophrenia) and disorders of movement control. Impulsivity usually has a negative connotation because of the harm it can cause not only to the impulsive individual but, through that individual's behaviour, to others. Impulsive acts are often preceded by a period of rising tension, which resolves into a sense of 


\section{Impact of Neurotransmitters on Health through Emotion}

relief and well-being once the act is completed. The role of dopamine in impulsive behaviour is most likely tied to its involvement in the brain's system for rewarding particular behaviours..

Dopamine interacts with multiple post synaptic receptors and is assumed to promote slightly different responses in the neuron it serves. Depressed individuals nearly always have low levels of serotonin in their central nervous systems. It is not surprising that impaired dopamine function could contribute to depression. The processing of negative emotions is said to be linked to the release of dopamine in the amygdale, prefrontal and medial temporal areas of the brain. In the central nervous system, high concentrations of dopamine are linked to love alongside attention, motivation and goal-directed behaviour. In addition, the ability to focus, remember, cherish of a beloved indicates that dopamine is involved in this phenomenon. Increased levels of dopamine had indeed been linked to undivided attention. High concentrations of dopamine in the brain had also been associated with euphoria, loss of appetite, hyperactivity, increased mental activity, less likely to feel fatigue, the lack of need to sleep, 'hyperactive fear-like state, anxiety and panic. Dopamine is a neurotransmitter involved in decision- making. It could be hypothesized that alteration of emotional responses in elderly subjects is mostly related to concomitant impairment of DA neurone activity. Intense pleasure experienced when listening to music is associated with dopamine activity in the mesolimbic reward system, including both dorsal and ventral striatum .

\section{GABA:}

GABA is involved in sedation, anxiety, and muscle relaxation and tonic inhibition. It acts at inhibitory synapses in the brain by binding to specific transmembrane receptors in the plasma membrane of both pre and post synaptic neuronal processes causing cell cycle arrest in the $\mathrm{S}$ phase, limiting growth.

Diseases Associated with GABA include focal epilepsy, which is decreased local GABAmediated inhibition. Many facets of epilepsy can be elicited experimentally by blocking GABA receptors with the toxin picrotoxin. The decrease in GABA inhibition permits cells to fire synchronously, thus producing massive local excitation and initiation of a seizure. Some finding suggests that some initial imbalance in the GABA ergic system may underlie aspects of this disorder.

\section{Glutamate :}

The neurotransmitter glutamate is highly toxic to neurons when present for extended periods. One of the best understood clinical conditions involving glutamate is neuronal injury following stroke or trauma. Derangements in glutamate metabolism or receptor activation have been implicated in a wide variety of pathologic conditions such as Alzheimer's and Huntington's chorea.

\section{Combined Effects:}

Together Serotonin, Nor adrenaline and Dopamine are involved in control of many mental states, sometimes acting on their own and other times acting together. Important features they share 
include cognitive function, mood, emotion, motivation, appetite, aggression, anxiety. Abnormality in their neurotransmitter activity results in many brain disorders like Parkinson's disease, schizophrenia, migraine, anxiety disorders and depressive psychosis. Nor epinephrine and Serotonin have been implicated to play an important role in sleep.

\section{CONCLUSION}

There are different types of neurotransmitters in the brain and each of them has their own effect on the human body. Most people have not heard of several common neurotransmitters, including serotonin, dopamine, nor epinephrine and epinephrine and are familiar with at least some of their functions in regards to mood and sleep.

\section{REFERENCES}

Barbar L Fredrickson and Christine Branignan, "Positive emotions broaden the scope of attention and thought-action repertoires,” Cognition and emotion, 2005, 19(3), 313-322

Bertram G Katzung, Susan B masters, "Basic and Clinical Pharmacology,” Nitric oxide Chapter 19, pp 334-336

H,P.Rang, M.M.Dale et.al, Autonomic nervous system, Rand and Dale’s Pharmacology, seventh edition, , Churchill Livingstone, USA, 2007, pg no:63,143,144.

Jagadish Prasad, “ Conceptual Pharmacology,” Chapter 4, pp 174

K Sembulingam, Prema Sembulingam, Neurotransmitters, Essentials of medical physiology, Sixth edition, Jaypee Brothers, New Delhi, 2013, Pg.No:787-791.

KD Tripathi, Autonomic nervous system, Essentials of Medical Pharmacology, Sixth Edition, Jaypee Brothers, New Delhi,2010,pg.no:89-92,95,96,119,153,163,164.

Pilar Salgado-Pineda et al, "Dopaminergic Contribution to the Regulation of Emotional Perception,” Clinical Neuropharmacology, Volume 28, Number 5, pp- 228- 237, September - October 2005

R Cools et al, Multiple functions of serotonin and dopamine, Neuropsychopharmacology, 2011, Pg. No: 36, 98-113.

William R Clark, “Are we hardwired? The role of genes in human behaviour,” Chapter 8 The role of neurotransmitters in human behaviour

\section{Website:}

http://nba.uth.tmc.edu/neuroscience/s1/chapter11.html

http://nba.uth.tmc.edu/neuroscience/s1/chapter12.html

http://nba.uth.tmc.edu/neuroscience/s1/chapter14.html

http://nba.uth.tmc.edu/neuroscience/s1/chapter13.html

http://psychology.wikia.com/wiki/Neurotransmitters. 